\title{
Deep brain stimulation-associated brain tissue imprints: a new in vivo approach to biological research in human Parkinson's disease
}

\author{
Affif Zaccaria ${ }^{1,9^{* \dagger}}$, Ali Bouamrani ${ }^{2 \dagger}$, Stephan Chabardès ${ }^{3,7}$, Michèle El Atifi ${ }^{2}$, Eric Seigneuret ${ }^{3}$, Johannes A. Lobrinus ${ }^{4}$, \\ Michel Dubois-Dauphin ${ }^{5}$, François Berger ${ }^{6}$ and Pierre R. Burkhard ${ }^{1,8}$
}

\begin{abstract}
Background: Deep brain stimulation (DBS) of the subthalamic nucleus (STN) or the internal segment of the globus pallidus (GPi) has been established as a highly effective symptomatic therapy for Parkinson's disease (PD). An intriguing biological aspect related to the DBS procedure is that a temporary contact establishes between surgical instruments and the surrounding brain tissue. In this exploratory study, we took advantage of this unique context to harvest brain material adhering to the stylet routinely used during surgery, and to examine the biological value of these samples, here referred to as "brain tissue imprints" (BTIs).
\end{abstract}

Results: Nineteen BTIs from 12 STN- or GPi-electrode implanted patients were obtained in vivo during DBS surgery, without any modification of the surgical procedure. Immunofluorescence analyses confirmed that our approach allowed the harvesting of many neural cells including neurons harboring distinct neurotransmitter markers. Shotgun proteomic and transcriptomic analyses provided for the first time molecular information from DBSassociated brain samples, and confirmed the compatibility of this new type of sample with poly-omic approaches. The method appears to be safe and results consistent.

Conclusions: We here propose BTIs as original and highly valuable brain samples, and DBS-related brain imprinting as a new conceptual approach to biological research in living patients with PD.

Keywords: Parkinson's disease, Deep Brain Stimulation, Brain Tissue Imprint, Histological analysis, Poly-omic approaches

\section{Background}

Nearly four decades of intensive research have proved yet unsuccessful at deciphering the aetiology and mechanisms underlying nigral decay in Parkinson's disease (PD) [1-3], in spite of myriads of hypotheses that have been proposed thus far [4]. Reasons for this ongoing uncertainty are many, including the extensive use of animal

\footnotetext{
* Correspondence: Affif.Zaccaria@unige.ch

François Berger and Pierre R. Burkhard are the co-last authors

${ }^{\dagger}$ Equal contributors

'NeuroProteomics Group, University Medical Center, Faculty of Medicine, Geneva, Switzerland

${ }^{9}$ Centre Medical Universitaire (CMU), Rue Michel Servet 1, CH-1211 Genève 4, Switzerland

Full list of author information is available at the end of the article
}

models only partly relevant to human PD [5] and the conducting of hypothesis-driven protocols $[6,7]$ that may be too narrow to disentangle the numerous mechanisms likely at work in PD degeneration. Because hypothesis-driven protocols based on animal models of PD have failed somehow, more global and hypothesisfree strategies have been undertaken in humans, possibly the only source of biological and molecular information truly relevant to PD [8]. Examples include genome-wide association studies $[9,10]$ or omic approaches [11-13]. In $\mathrm{PD}$, while various peripheral tissues or biological fluids are easily amenable to collection in living patients, relevant neural tissue can be obtained only at autopsy from deceased patients. Indeed, during the last ten years 
or so, a number of proteomic and transcriptomic studies have been conducted on post-mortem brain tissue samples from various PD-relevant brain structures [11, 14]. However, post-mortem samples are associated with a number of limiting and confounding factors [15-17]. First, difficulties to obtain autopsy tissue [18] tend to keep the number of available samples per study quite low, thus minimizing statistical power to draw firm conclusions. Second, the quality of autopsy tissue is intimately related to the duration and specificities of the premortem agonal state and post-mortem interval (PMI) $[19,20]$. Finally, with few exceptions, post-mortem samples are obtained from patients at an advanced stage of disease, making it difficult to identify early molecular events at the basis of the complex basal ganglia (BG) electrophysiology and PD pathogenesis.

To overcome these limitations, and considering all issues discussed above, it may seem that the ultimate sample for biological research in human PD is still to be found. Ideally, it has to be safely and routinely obtained from a large number of living individuals with welldocumented sporadic PD. It should consist of biological material directly involved in PD pathogenesis, in this case brain material. Methods to collect samples should be standardized and sampling-to-freezer time should be kept as short as possible.

While this may seem unrealistic at first glance, we propose a novel strategy to obtain PD-relevant samples that may approximate these requirements, based on the concept of in vivo brain tissue imprinting during electrode implantation for deep brain stimulation (DBS) [21]. In fact, we hypothesized that, during this procedure, brain material of interest may adhere to surgical instruments, which transiently interact with implanted nuclei and can be retrieved and analysed at the cellular and molecular level, for example through omic strategies. In this study, we demonstrate for the first time the feasibility, safety, and scientific interest of collecting and studying such DBS-associated samples, here referred to as brain tissue imprints (BTIs), which may provide unique biological signatures of deep brain structures, and, in our opinion, may represent a new paradigm in PD research using highly relevant brain material from living patients.

\section{Results}

\section{Brain tissue imprinting of BG nuclei}

In this study, 12 patients have been offered bilateral electrode implantation [22] in STN or GPi to alleviate their motor symptoms (Table 1). Once the optimal target site for the chronic electrode to be implanted has been defined, neurosurgeon A or B lowered a blunt stylet inside a guiding tube (Fig. 1a), to the neuroimaging-defined target site (Fig. 1b). First, the radiographic projection on the patient's preoperative brain MRI confirmed that the stylet tip was within the surgical target, i.e. the STN or GPi (Fig. 1c). The surgeon kept the stylet tip exposed

Table 1 Patient information and clinicopathological data of human BTIs

\begin{tabular}{|c|c|c|c|c|c|c|c|}
\hline Patients & Gender & Age, yr & Disease duration, yr & Implanted nuclei & Neurosurgeon & BTI number Left (L) or Right (R) & Experiment \\
\hline \multirow[t]{2}{*}{ P1 } & $\mathrm{F}$ & 41 & 6 & STN & A & L1 & SEM \\
\hline & & & & & & R1 & $\mathrm{CB}-\mathrm{HHC}$ \\
\hline \multirow[t]{2}{*}{ P2 } & $\mathrm{F}$ & 60 & 10 & STN & B & L2 & CB-IHC \\
\hline & & & & & & R2 & $\mathrm{CB}-\mathrm{HHC}$ \\
\hline \multirow[t]{2}{*}{ P3 } & M & 52 & 8 & STN & A & L3 & $P$ \\
\hline & & & & & & R3 & $\mathrm{T}$ \\
\hline \multirow[t]{2}{*}{ P4 } & M & 53 & 7 & STN & B & L4 & $\mathrm{T}$ \\
\hline & & & & & & R4 & T \\
\hline P5 & M & 46 & 6 & STN & A & L5 & $P$ \\
\hline P6 & M & 54 & 11 & STN & B & R6 & $P$ \\
\hline \multirow[t]{2}{*}{ P7 } & M & 61 & 9 & STN & B & L7 & P \\
\hline & & & & & & R7 & $P$ \\
\hline \multirow[t]{2}{*}{ P8 } & $M$ & 53 & 9 & GPi & B & L8 & P \\
\hline & & & & & & R8 & $P$ \\
\hline P9 & $\mathrm{F}$ & 45 & 10 & GPi & A & L9 & P \\
\hline P10 & $M$ & 69 & 14 & GPi & $A$ & L10 & $P$ \\
\hline P11 & $\mathrm{F}$ & 59 & 12 & GPi & A & L11 & P \\
\hline P12 & $M$ & 52 & 11 & GPi & B & R12 & $P$ \\
\hline
\end{tabular}

Eight men (M) and 4 women (F) had been offered DBS in STN or GPi by neurosurgeon A or B. BTIs were obtained from left (L) or right (R) hemisphere and used for scanning electron microscopy (SEM), cellblock immunohistochemical (CB-IHC), proteomic (P) or transcriptomic (T) analyses 


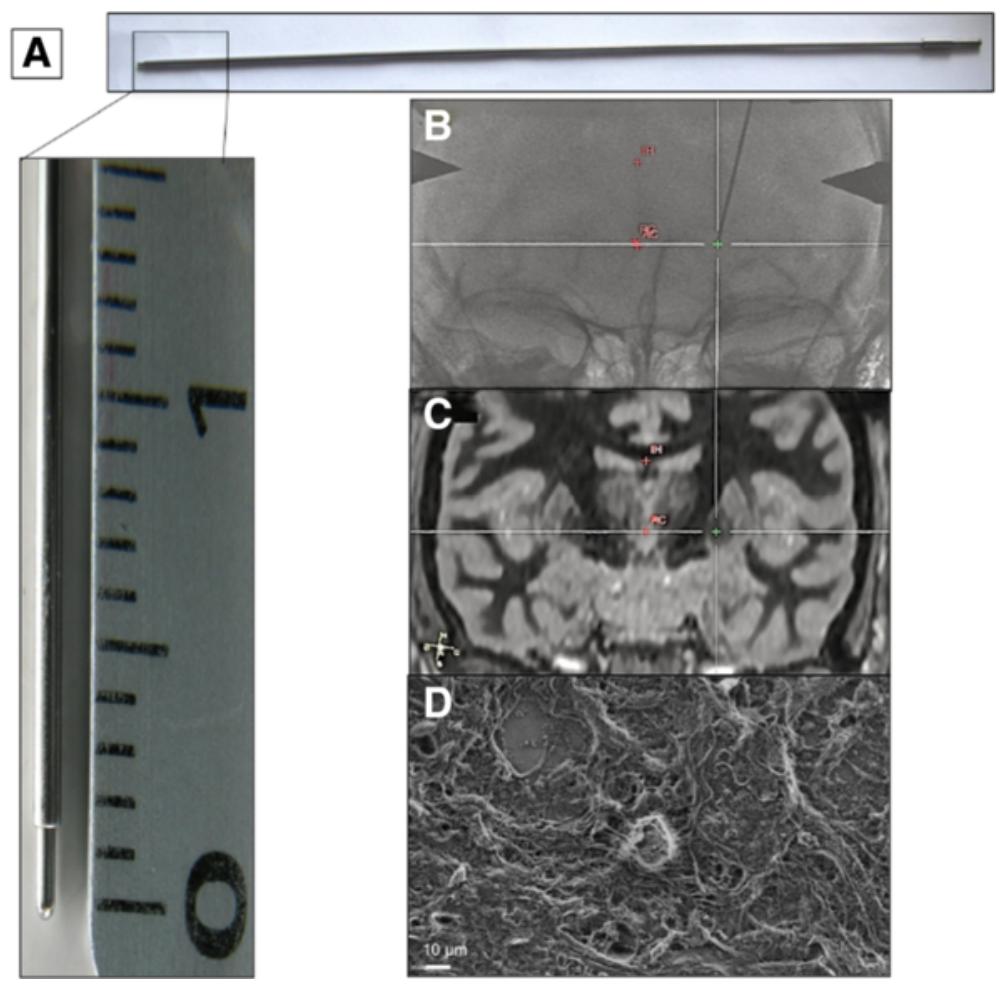

Fig. 1 The BTI procedure. a The set combines a guide tube and a rigid blunt stylet. The stylet slides through the guide tube and its tip protrudes by $1.5 \mathrm{~mm}$ out of the guiding tube. b-c The stylet tip is lowered to the targeted nucleus (here the GPi) and interacts with the brain parenchyma according to $(\mathbf{b})$ the perioperative radiographic control and (c) the pre-operative MRI-defined trajectory. $\mathbf{d}$ Scanning electron microscopy of the stylet tip-associated tissue revealed the presence of cell bodies and fibers

one minute into the patient brain before withdrawing it through the guide tube, and finally removed the set from the brain parenchyma. From the 12 bilaterally implanted patients, a total of 19 stylets was collected and tips that harvested tissue fragments as observed with scanning electron microscopy (Fig. 1d) were immersed in appropriate buffer for further analyses (Table 1). No immediate or late complication from the procedure could be identified. These data demonstrate that the transient contact of the stylet tip with DBS targeted nuclei allows brain material harvesting with no significant modification of the routine DBS procedure.

\section{Confirming the capture of neural cells by immunohistochemical analyses}

To characterize these tissue fragments and confirm the capture of neural cells, three different STN-associated stylet tips were collected in fixative solution for immunohistochemical analyses. First, a hematoxylin-eosin staining revealed the presence of numerous cells in these tissue fragments, variably clustered throughout the sample (Additional file 1: Figure S1). The neuronal and glial nature of these cells was confirmed by immunohistochemical analyses using $\beta 3$-Tubulin or NeuN, and GFAP monoclonal antibody, respectively (Fig. 2). In fact, $\beta 3$ -
Tubulin (Fig. 2a) and NeuN (Fig. 2B and Additional file 1: Figure S1) staining from $10 \mu \mathrm{m}$-thick sections revealed the presence of many positive cells whereas GFAP staining (Fig. 2b and Additional file 1: Figure S1) highlighted an important astroglial network. In addition, V-GLUT1 (Fig. 2e) and GAD67 (Fig. 2f) immunostaining demonstrated the presence of glutamatergic and GABA-ergic neurons, respectively, and we also observed the presence of large $\mathrm{TH}$-immunoreactive patches lacking nuclei.

These experiments confirm that DBS-obtained tissue fragments contain, besides astrocytes, neurons and fibers harbouring various neurotransmitter markers and reflect brain tissue of BG origin. We defined this collected material as brain tissue imprints (BTIs).

\section{Quantifying extracted proteins from STN and GPi- associated BTIs}

Before to envisage potential proteomic studies, it appeared important to determine the BTI-collected protein quantity, which, if too low, could be a limiting factor of our approach. For this purpose, 12 blunt stylets that were lowered in the STN $(n=6)$ or GPi $(n=6)$ by neurosurgeon A or B (Table 1) were collected. First, the Bradford protein assay of the 12 samples varied from 6.8 to $17.5 \mu \mathrm{g}$ of proteins with a mean capture of $10.2 \pm 3.6 \mu \mathrm{g}$ 

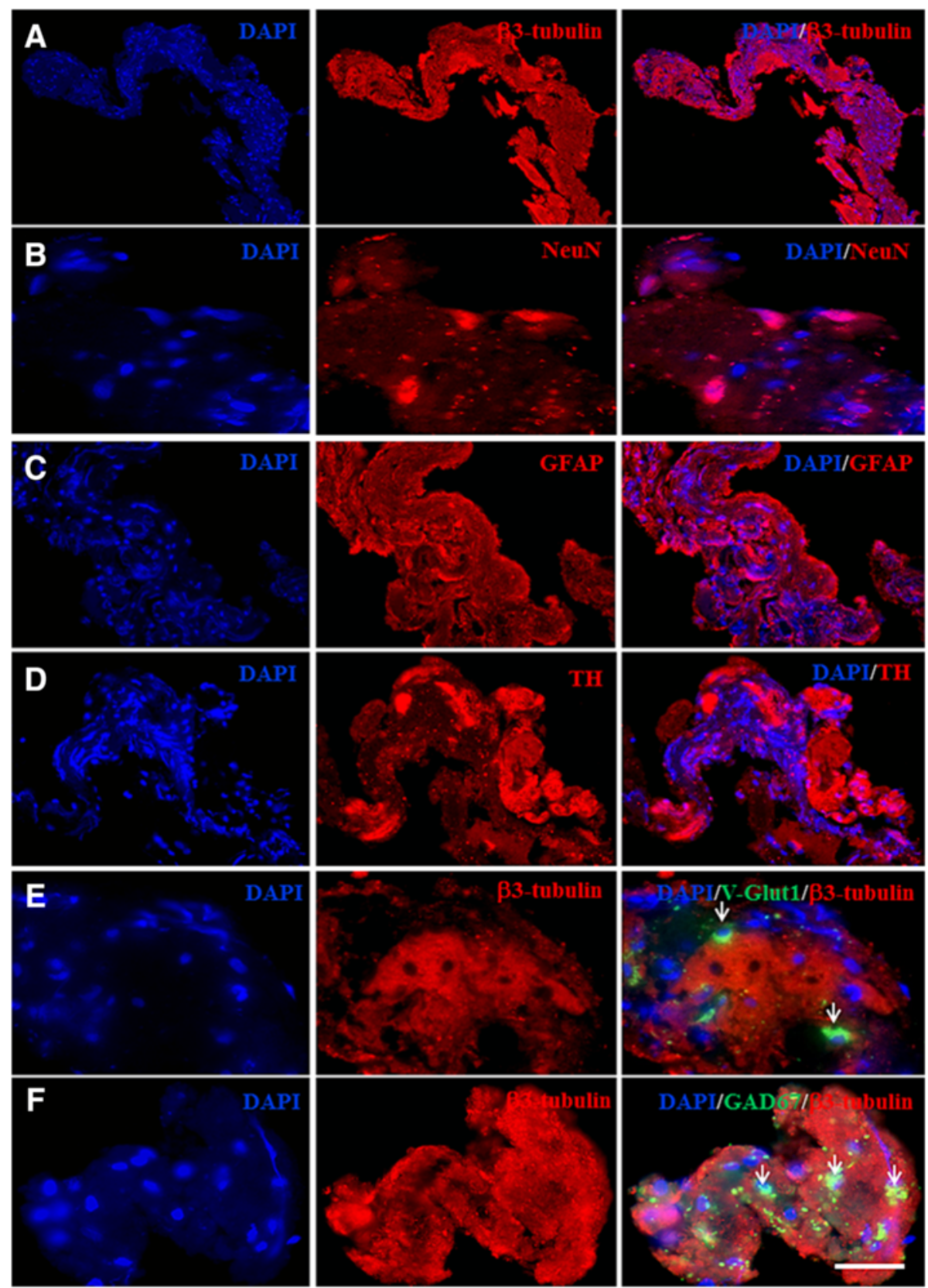

Fig. 2 BTI-associated immunohistochemical analyses. Neuronal markers $\beta 3$-tubulin (a) and NeuN (b) as well as astroglial marker GFAP (c) were identified in BTIs. The presence of tyrosin hydroxylase $(\mathrm{TH})$ was detected in regions poor in cell nuclei, suggesting the presence of TH-immunoreactive axons (d). Glutamatergic (e) and GABA-ergic (f) $\beta 3$-tubulin immunoreactive neurons were also detected (arrows). Scale bar: $A=160 \mu \mathrm{m}, \mathrm{B}=$ $20 \mu \mathrm{m}, \mathrm{C}=90 \mu \mathrm{m}, \mathrm{D}=85 \mu \mathrm{m}, \mathrm{E}$ and $\mathrm{F}=30 \mu \mathrm{m}$

of proteins. The comparison between STN and GPiassociated BTIs revealed no statistical difference between he different nuclei with $11.4 \pm 4.4$ and $8.9 \pm 2.4 \mu \mathrm{g}$ respectively (Mann Whitney test, $p=0.47$ ) (Fig. 3a). In addition, we did not observe any significant difference in extracted-protein quantity according to the neurosurgeon who collected the BTIs. In fact, BTIs revealed $10.8 \pm 4.1$ and $9.6 \pm 3.3 \mu \mathrm{g}$ of proteins for neurosurgeon $\mathrm{A}$ and $\mathrm{B}$, respectively (Mann Whitney test, $p=0.78$ ), (Fig. 3a). These results suggest that BTIs can be consistently obtained in any electrodeimplanted nucleus, are neurosurgeon-independent and contain amounts of proteins suitable for any proteomic protocols. 


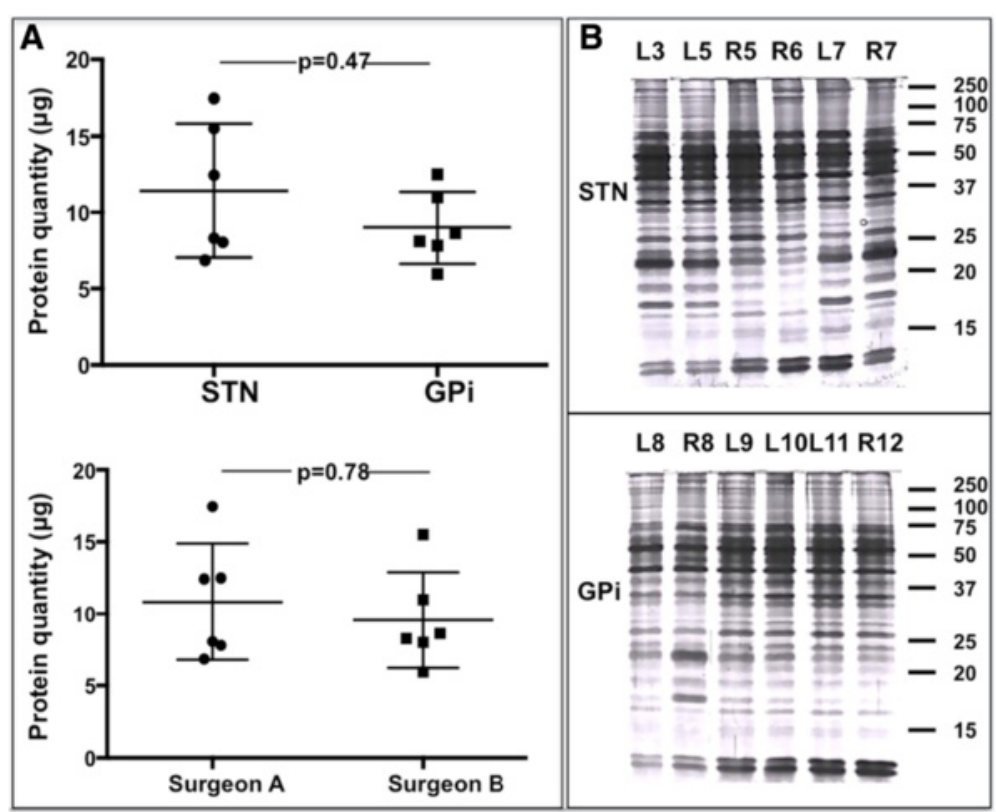

Fig. 3 a BTI-extracted protein quantity. Scatter plot showing the quantity of proteins extracted from BTIs according to the targeted nucleus (up) and to the surgeon (bottom). b 1D SDS-PAGE electrophoresis of STN (top) and GPi (bottom) -associated BTls. Two micrograms of proteins were loaded on $15 \%$ polyacrylamide gel and revealed by silver staining

\section{Examining the detailed protein content of STN and GPi- associated BTls by shotgun proteomics}

To obtain a qualitative protein profile of these imprints, we separated by 1D electrophoresis both STN and GPiassociated BTIs (Fig. 3b). First, all samples revealed complex profiles with the presence of many protein bands on a wide mass range, from 14 to $250 \mathrm{kDa}$. Moreover, for both nuclei, the different BTIs revealed similar qualitative profiles with the presence of the same protein bands. Finally the comparison of STN and GPi-associated BTIs did not reveal obvious significant differences in protein profiles, showing a reproducible overall protein pattern. To further characterize STN and GPI-associated BTIs, 1D SDSPAGE fractionation was combined with nano-LC-MS/MS analysis for extensive protein identification. Nano-LC-MS/ MS analysis of STN-associated BTIs allowed the identification of 1'081 different proteins. The analysis of GPIassociated BTIs revealed 894 different proteins. Combining both protein lists highlighted a total of 1'298 distinct proteins (Additional file 2: Table S1). Among the 1'298 identified proteins, we observed neuron, astrocyte and oligodendrocyte-enriched proteins such as neuronal growth regulator 1, astrocytic phosphoprotein PEA-15 and myelin-oligodendrocyte glycoprotein (Table 2). We also identified proteins implicated in different nervous cell functions such as cell architecture, cell adhesion, synapse organization or neural maturation. Finally, consistent with immunofluorescence data detailed above, we also observed neurotransmitter-associated receptors and transporters such as glutamate receptor 2, metabotropic glutamate receptor 3 and vesicular glutamate transporter 1 . These results confirmed the suitability of BTI to explore in depth the structural and functional proteome of different electrode-implanted nuclei.

\section{Functional analysis of BTI-identified proteins}

To investigate the functional role of BTI-identified proteins, we separately submitted STN and GPi protein lists to Ingenuity Pathway Analysis Software (IPA) [23]. This automated annotation tool uses a knowledge database and assigns proteins to functional classes or specific canonical pathways related to various biological processes. To confirm the relevance of our BTI approach, we determined the relative representation of neurological diseases and brain-associated signalling pathways in STN and GPi-associated BTIs, respectively. First, the functional analysis revealed neurological diseases and psychological disorders as the two most represented disorders in both types of BTIs (Fig. 4a). In fact, STN and GPi protein lists respectively revealed 412 and 381 different species associated to neurological disease pathways, which represented 38.1 and $42.6 \%$ of the total identified proteins in STN and GPi, respectively. Moreover, among these neurological disease-associated proteins, 69 and 56 species, which represented 6.4 and $6.3 \%$ of the total identified proteins, were associated to PD in STN and GPi-BTIs, respectively. Finally, we also observed that canonical pathways (CPs), which were specific to brain functionality such as synaptic long term potentiation 
Table 2 Brain-enriched and PD-associated proteins from BTls. Table listing the accession number in Unirot database (ID), the isoelectric point (pl), the molecular weight (protein mass), and the name (description) of different brain-enriched or PD-associated proteins (bold)

\begin{tabular}{|c|c|c|c|c|}
\hline $\begin{array}{l}\text { Prot } \\
\text { number }\end{array}$ & ID & $\begin{array}{l}\text { Prot } \\
\mathrm{pl}\end{array}$ & $\begin{array}{l}\text { Protein } \\
\text { mass (Da) }\end{array}$ & Description \\
\hline 1 & PEA15_HUMAN & 4.9 & 15088 & $\begin{array}{l}\text { Astrocytic phosphoprotein } \\
\text { PEA-15 }\end{array}$ \\
\hline 2 & BASP1_HUMAN & 4.6 & 22562 & Brain acid soluble protein 1 \\
\hline 3 & CNRP1_HUMAN & 5.2 & 14224 & $\begin{array}{l}\text { CB1 cannabinoid receptor- } \\
\text { interacting protein } 1\end{array}$ \\
\hline 4 & GFAP_HUMAN & 5.5 & 49880 & Glial fibrillary acidic protein \\
\hline 5 & GRIA2_HUMAN & 7.6 & 96183 & Glutamate receptor 2 \\
\hline 6 & PEBP1_HUMAN & 7.8 & 21057 & $\begin{array}{l}\text { Hippocampal cholinergic } \\
\text { neurostimulating peptide }\end{array}$ \\
\hline 7 & LSAMP_HUMAN & 5.7 & 31818 & $\begin{array}{l}\text { Limbic system-associated } \\
\text { membrane protein }\end{array}$ \\
\hline 8 & LYNX1_HUMAN & 6.6 & 11937 & $\begin{array}{l}\text { Ly-6/neurotoxin-like } \\
\text { protein } 1\end{array}$ \\
\hline 9 & GRM3_HUMAN & 7.7 & 96386 & $\begin{array}{l}\text { Metabotropic glutamate } \\
\text { receptor } 3\end{array}$ \\
\hline 10 & MBP_HUMAN & 11.5 & 21493 & Myelin basic protein \\
\hline 11 & MYPR_HUMAN & 8.8 & 29946 & Myelin proteolipid protein \\
\hline 12 & MOG_HUMAN & 9.0 & 25110 & $\begin{array}{l}\text { Myelin-oligodendrocyte } \\
\text { glycoprotein }\end{array}$ \\
\hline 13 & NCAM1_HUMAN & 4.8 & 92408 & $\begin{array}{l}\text { Neural cell adhesion } \\
\text { molecule } 1\end{array}$ \\
\hline 14 & NCAM2_HUMAN & 5.5 & 91052 & $\begin{array}{l}\text { Neural cell adhesion } \\
\text { molecule } 2\end{array}$ \\
\hline 15 & L1CAM_HUMAN & 5.8 & 137831 & $\begin{array}{l}\text { Neural cell adhesion } \\
\text { molecule L1 }\end{array}$ \\
\hline 16 & NCALD_HUMAN & 5.4 & 22114 & Neurocalcin-delta \\
\hline 17 & NCAN_HUMAN & 5.2 & 140732 & Neurocan core protein \\
\hline 18 & NCDN_HUMAN & 5.4 & 77243 & Neurochondrin \\
\hline 19 & NFASC_HUMAN & 6.6 & 130112 & Neurofascin \\
\hline 20 & NFH_HUMAN & 5.8 & 105639 & $\begin{array}{l}\text { Neurofilament heavy } \\
\text { polypeptide }\end{array}$ \\
\hline 21 & NFL_HUMAN & 4.6 & 61385 & $\begin{array}{l}\text { Neurofilament light } \\
\text { polypeptide }\end{array}$ \\
\hline 22 & NFM_HUMAN & 4.9 & 102341 & $\begin{array}{l}\text { Neurofilament medium } \\
\text { polypeptide }\end{array}$ \\
\hline 23 & NEUM_HUMAN & 4.7 & 24803 & Neuromodulin \\
\hline 24 & NRCAM_HUMAN & 5.7 & 130668 & $\begin{array}{l}\text { Neuronal cell adhesion } \\
\text { molecule }\end{array}$ \\
\hline 25 & NEGR1_HUMAN & 6.5 & 31426 & Neuronal growth regulator 1 \\
\hline 26 & GPM6A_HUMAN & 5.7 & 29905 & $\begin{array}{l}\text { Neuronal membrane } \\
\text { glycoprotein M6-a }\end{array}$ \\
\hline 27 & GPM6B_HUMAN & 5.9 & 36220 & $\begin{array}{l}\text { Neuronal membrane } \\
\text { glycoprotein M6-b }\end{array}$ \\
\hline 28 & SEPT3_HUMAN & 6.5 & 40100 & Neuronal-specific septin-3 \\
\hline 29 & NPTN_HUMAN & 7.6 & 37792 & Neuroplastin \\
\hline 30 & NTRI_HUMAN & 6.0 & 31738 & Neurotrimin \\
\hline 31 & SYN1_HUMAN & 9.9 & 70033 & Synapsin-1 \\
\hline 32 & SYN2_HUMAN & 8.7 & 62847 & Synapsin-2 \\
\hline 33 & SYN3_HUMAN & 9.5 & 63303 & Synapsin-3 \\
\hline
\end{tabular}

Table 2 Brain-enriched and PD-associated proteins from BTIs. Table listing the accession number in Unirot database (ID), the isoelectric point (pl), the molecular weight (protein mass), and the name (description) of different brain-enriched or PD-associated proteins (bold) (Continued)

\begin{tabular}{|c|c|c|c|c|}
\hline 34 & SV2A_HUMAN & 5.4 & 82695 & $\begin{array}{l}\text { Synaptic vesicle } \\
\text { glycoprotein } 2 \mathrm{~A}\end{array}$ \\
\hline 35 & SNG3_HUMAN & 8.8 & 24555 & Synaptogyrin-3 \\
\hline 36 & SYNJ1_HUMAN & 7.1 & 143254 & Synaptojanin-1 \\
\hline 37 & SYPH_HUMAN & 4.7 & 33845 & Synaptophysin \\
\hline 38 & SNP25_HUMAN & 4.7 & 23315 & $\begin{array}{l}\text { Synaptosomal-associated } \\
\text { protein } 25\end{array}$ \\
\hline 39 & SYT1_HUMAN & 8.4 & 47573 & Synaptotagmin-1 \\
\hline 40 & VGLU1_HUMAN & 7.6 & 61613 & $\begin{array}{l}\text { Vesicular glutamate } \\
\text { transporter } 1\end{array}$ \\
\hline 41 & VAMP1_HUMAN & 8.7 & 12658 & $\begin{array}{l}\text { Vesicle-associated } \\
\text { membrane protein } 1\end{array}$ \\
\hline 42 & VAMP2_HUMAN & 8.9 & 12532 & $\begin{array}{l}\text { Vesicle-associated } \\
\text { membrane protein } 2\end{array}$ \\
\hline 43 & GMFB_HUMAN & 5.3 & 16582 & Glia maturation factor beta \\
\hline 44 & SYNPO_HUMAN & 9.4 & 73666 & Synaptopodin \\
\hline 45 & SYUG_HUMAN & 4.9 & 13330 & Gamma-synuclein \\
\hline 46 & SYUB_HUMAN & 4.5 & 14288 & Beta-synuclein \\
\hline 47 & SYUA_HUMAN & 8.8 & 11371 & Alpha-synuclein \\
\hline 48 & PARK7_HUMAN & 6.8 & 19891 & Protein DJ-1 \\
\hline 49 & UCHL1_HUMAN & 5.5 & 24824 & $\begin{array}{l}\text { Ubiquitin carboxyl } \\
\text { terminal hydrolase } \\
\text { isozyme L1 }\end{array}$ \\
\hline 50 & VPS35_HUMAN & 5.4 & 91707 & $\begin{array}{l}\text { Vacuolar protein } \\
\text { sorting-associated pro- } \\
\text { tein } 35\end{array}$ \\
\hline
\end{tabular}

and neurotransmitter signalling pathways, or described as altered in PD such as mitochondrial dysfunction, oxidative phosphorylation and axonal guidance associated pathways were significantly represented ( $p$-value $<0.05)$ in both types of BTIs (Fig. 4b). Of particular interest, PD signalling was significantly represented in both type of BTIs with the identification of several PD-relevant proteins such as $\alpha$ synuclein, DJ-1 protein, UCHL1 protein and VPS35 protein, within which mutations have been associated with autosomal dominant or recessive forms of PD (Table 2). In addition, the proportion of proteins that was associated to these CPs were quite similar between the two types of BTIs. These results demonstrate the ability of the BTI approach to highlight brain and PD-related functional signatures in different nuclei and confirm that proteomics analysis of BTIs could be an appropriate strategy to study PD altered signalling pathways.

\section{Confirming the extraction of high-quality RNA from STN- associated BTIs}

Given the major interest of gene expression profiling in PD-associated research, we assessed the capacity of BTIs 


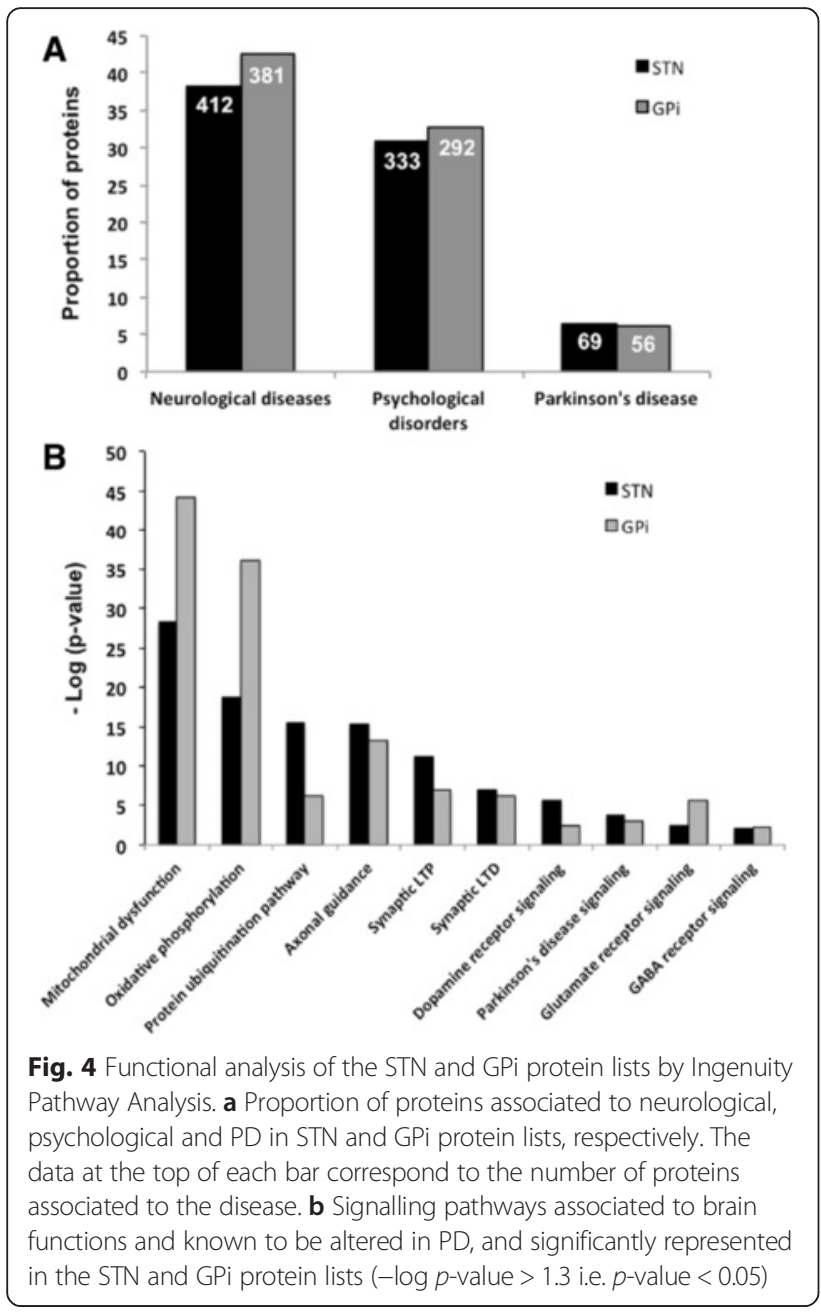

to extract good quality RNAs from in vivo STN nucleus in PD patients. For this purpose, three different STNstylets were collected, total RNA was extracted from these BTIs, and we applied a double amplification protocol to generate sufficient biological material for further analysis. After amplification rounds, these three BTIs revealed between 65 and $200 \mu \mathrm{g}$ of RNAs, i.e. quantities appropriate for hybridization microarray analysis. To confirm the good quality of BTI-extracted RNAs, we applied the same preparation protocol on brain cortical sections obtained during epilepsy surgery and used as high-quality control sample. For this control sample, given the availability of sufficient starting material, the RNA quality was controlled and confirmed before amplification by capillary electrophoresis migration, which revealed full length RNAs and RNA integrity number (RIN) of 8.3 (Additional file 3: Figure S2). After a double amplification round, the control sample naturally revealed electrophoretic profile with shorter products that we compared with the BTI-obtained profiles. As shown on Fig. 5a, we observed a great similarity between BTI and control electrophoretic profiles with same length RNAs, implying that similarly to the control sample, BTI-collected RNAs were also full length and intact before amplification. These results demonstrate that high quality RNAs can be retrieved in vivo from DBS-targeted nuclei and are suitable for microarray analyses.

Given the sufficient quantity and the good quality of extracted RNAs, the samples were separately hybridized on microarrays to determine the complexity of expressed transcripts in BTI samples. Detailed results are available in Additional file 4: Table S2. The analysis of BTIextracted RNAs highlighted from 29'842 to 35'701 different detected probe sets, which corresponded to a chip hybridization rate from 54.5 to $65 \%$ (Fig. 5b). A chip hybridization rate of $60 \%$ was obtained for the control sample, the analysis of which revealed 33'073 detected probe sets. Moreover the qualitative analysis of the BTIassociated chips revealed 26'112 common detected probe sets in the different BTIs, suggesting a robust qualitative reproducibility of the BTI approach. Moreover intensities of these common detected probe sets were strongly correlated between the different samples (Fig. 5c). In fact, the graphical representation of the 26 '112 probe sets intensities in the different BTIs revealed a Pearson's correlation coefficient of 0.8 to 0.9 with a slope value between 0.97 and 1.0, suggesting that most of these common probe sets were similarly expressed in the different BTIs, which originated from the same type of brain structure in the same disease condition. Altogether, these results demonstrate that high quality transcriptomic analyses may be undertaken from our BTI approach.

\section{Discussion}

Accessing in vivo human brain samples for PDassociated research has long been considered an unrealistic approach, yet it may be one of the most relevant strategies to address such pivotal issues as etiopathogenic events, molecular pathways and diagnostic or therapeutic biomarkers. Since its first use in the ventral intermediate nucleus of the thalamus by Benabid et al. [24], DBS in PD has been extended to STN [25], GPi [26] and other deep brain nuclei [27], and has become the mainstay of surgical therapies to manage motor symptoms at early to advanced stages of the disease.

In the present study, we demonstrate for the first time that DBS-associated electrode implantation offers a unique opportunity to obtain in vivo fresh tissue imprints from relevant and electrophysiologically active deep brain structures. In fact, the stylet that is systematically used in DBS surgery, the tip of which is in immediate contact with the surrounding tissue, allows to recover, irrespective of brain targets and surgeons, tissue micro fragments that we have defined as BTIs. This unconventional material seems to be highly informative 


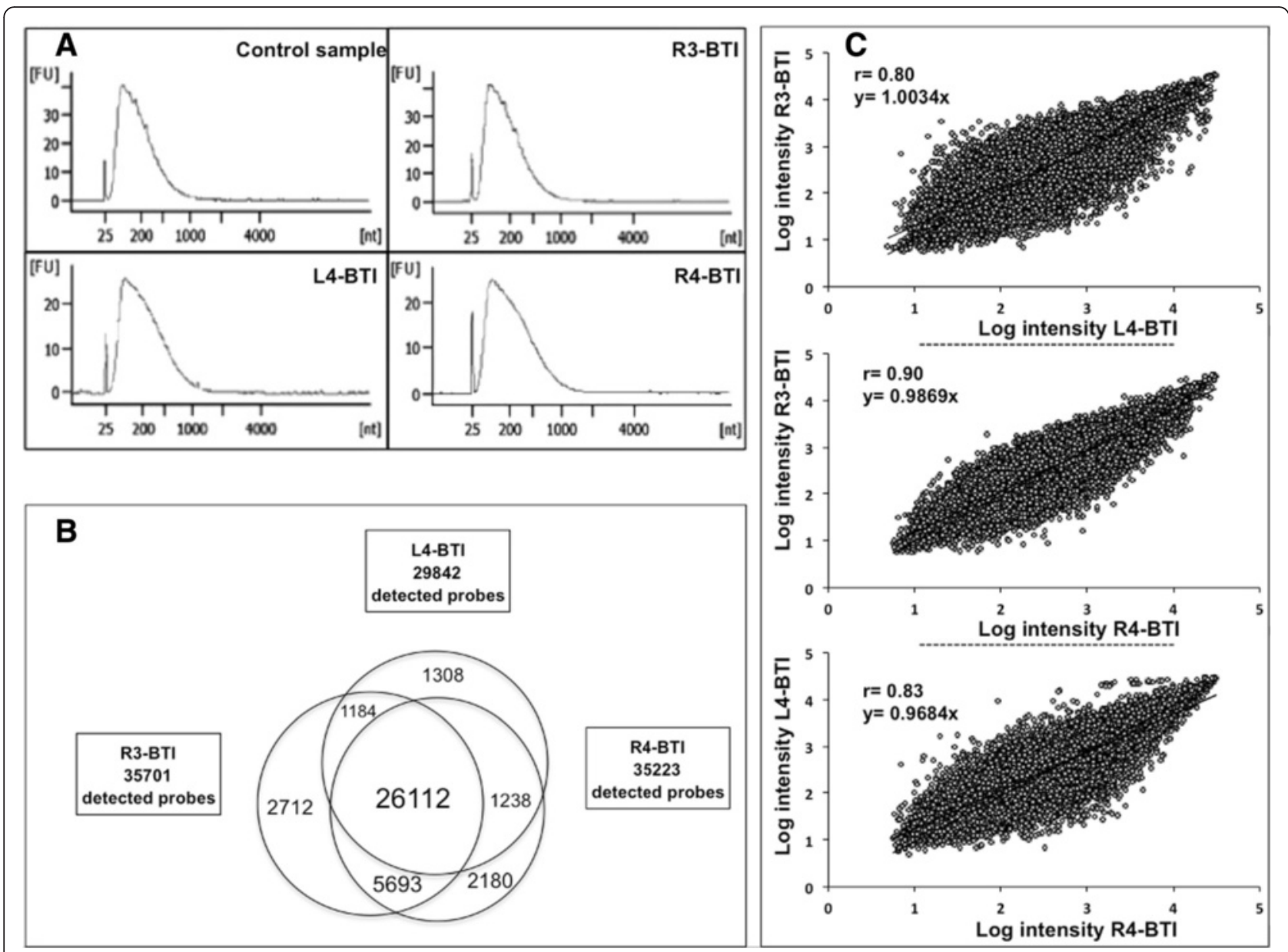

Fig. 5 Transcriptomic analysis of BTI-extracted RNAs. a Quality control of BTI-extracted RNA by capillary electrophoresis migration. The electrophoretic profiles of the BTI-samples were compared with a high-quality control sample. $\mathbf{b}$ Microarray hybridization of the BTIs. Venn diagrams representing the number of detected and common probe sets in BTI samples by microarray analysis. $\mathbf{c}$ Intensity correlation for the common detected probe sets in the 3 BTIs. Linear regression curve representing the intensity of expression of common probe sets in the three BTIs. The coefficient of correlation (r) and the coefficient of the slope is dysplayed on the graphe

and of great scientific interest. Indeed, immunohistological analyses of STN-derived BTIs consistently showed populations of glutamatergic and GABAergic neurons, and the presence of TH-immunoreactive fibers, as expected in the STN-SN region [28]. Whereas the SN pars reticulata mainly contains large GABAergic projecting neurons, the STN is populated with glutamatergic neurons, some of which collateralizing locally and some receiving $\mathrm{TH}$ projections from $\mathrm{SNpc}$-associated dopaminergic neurons [29]. Our findings are therefore in accordance with the current anatomical knowledge of these structures. Moreover, omic analyses of BTIs allowed to profile extensively their proteome and transcriptome, yielding lists of proteins and transcripts that turned out to be highly relevant for neurological diseases in general and PD in particular. Altogether, these preliminary results strongly support the concept of brain tissue imprinting during DBS surgery and the potential of this unique brain material to undergo informative poly-omic analyses.

The research paradigm proposed in this study is new and demonstrates many advantages over more conventional methods using post-mortem samples. First, the method of collecting BTIs is convenient and simple to perform in the context of DBS surgery, as it does not modify the DBS procedure. Second, this strategy proposes an in vivo access to deep brain nuclei and the collection of samples that are immediately processed in the surgical room within minutes after being captured, thus minimizing protein or RNA degradation $[19,20]$. Moreover, BTIs are obtained in awaken and off-medication patients, according to the routine DBS procedure, thus limiting the potentially deleterious influence of anaesthesia and antiparkinsonian medication, respectively, on molecular metabolism. Third, BTIs being collected from highly selective 
brain structures, in this particular case BG nuclei, seem particularly relevant to address functional and structural issues related to the underlying pathology. As far as PD is concerned, electrode implantation usually occurs 10 to 12 years after PD diagnosis, and performing BTIs in this context would ensure the access to a homogeneous group of PD brains at an earlier stage of disease than postmortem samples, which often derived from patients diagnosed 15 to 25 years prior to death. Furthermore, the recent use of DBS much earlier in the disease course, 5 to 7 years after diagnosis [30], opens the door to BTI-derived studies on molecular changes occurring only a few years after PD has started. Also, as DBS is currently exploring many other indications, including epilepsy, Alzheimer's disease, obsessive-compulsive disorders, Gilles de la Tourette syndrome, depression, and many others, this approach may likely be transposed to any DBS-treated neurological [31] or psychiatric diseases [32]. Fourth, another advantage of our approach involves the number of samples that could be collected, which is virtually unlimited, as opposed to post-mortem samples which are notoriously difficult to obtain. Furthermore, if various conditions are operated, stratification of samples according to pathologies or nuclei, and statistically powerful comparisons of results across groups, one serving as control for the other may become possible.

The BTI method proposed here is in a preliminary phase and may have some limitations, yet solutions to circumvent them can be already considered. First, the biological material composing BTI samples is likely heterogeneous, involving various cell types, neuronal populations, dendrites, axons and other cellular extensions, synapses and other contacts between cells, blood vessels, extracellular fluid and so on, thus lacking cellular or subcellular specificity. While this may seem problematic, it may also be beneficial in providing a global view of the molecular state of the structure considered at a certain time point. Second, only existing surgical tools were used in this study, with the purpose of not modifying the routine procedure of DBS, yet it is possible that BTIs were contaminated by unwanted material during the descent of the stylet. In the future, new BTI-specific instruments and protocols may be designed to achieve an even more selective imprint and high-grade BTI purity. Third, whereas molecular analysis of STN and GPiobtained BTIs may be of interest to investigate functional changes occurring in PD, other structures bearing alpha-synuclein pathology, like the $\mathrm{SNpc}$, might be more relevant to address pathogenesis. Indeed, because the SN lies anatomically just below the STN, it is not unusual for the tip of the DBS electrode to be placed within the SN [33], and we therefore assume that BTIs can also be captured from this pivotal structure in certain patients.
Finally, at variance with post-mortem samples that can be collected both in diseased and normal states, DBSassociated BTIs can only be obtained in pathological, DBS-treatable conditions and will never concern healthy patients. To circumvent this important limitation, we propose to use a variety of non-degenerative conditions where DBS targeting of the same structures is also indicated. For example, PD-associated BTIs from STN, GPi or VIM may be compared with matching BTIs from non-PD conditions such as OCD and epilepsy (STN), dystonia and Gilles de la Tourette syndrome (GPi), and essential tremor (VIM) respectively [34-38]. Moreover, the application of DBS in PD patients with different disease durations, from 5 to 15 years may enable the stratification of BTIs according to disease duration and disease stages, and the comparison of early versus late biological changes potentially occurring in the basal ganglia. Finally, as PD is an asymmetrical disease and as BTIs could be collected bilaterally, comparisons may involve less versus more affected basal ganglia structures for each patient. Thus, several options are available to obtain comparative samples. While they may not totally fulfil the definition of control samples, they are likely to be sufficiently distinct from PD to allow robust and meaningful comparative analyses to be performed.

\section{Conclusion}

In conclusion, this exploratory, proof-of-concept study proposes in vivo DBS-associated brain tissue imprinting as an original paradigm to explore the cellular and molecular profile of deep brain nuclei in human PD, and likely in any degenerative and non-degenerative DBS-treated neurological and psychiatric conditions. In particular, the biological material obtained through this approach appears to overcome most of the limitations inherent to post-mortem tissue and to be particularly suitable for omic research. Taking into account these aspects, BTI may become the ultimate sample of biological brain research in humans and significantly impact on future studies on pathogenesis, pathophysiology, biomarkers or therapeutic interventions in various neurological disorders.

\section{Methods \\ Study participants}

Twelve patients have been offered bilateral STN $(n=7)$ or GPi $(n=5)$ electrode implantation to alleviate their motor symptoms at the University Hospital of Grenoble. The STN-implanted group was 41 to 61 years (mean: $52.4 \pm 7.1$ years) and included five men and two women. The GPi-implanted group was 45 to 69 years (mean: $55.6 \pm 8.9$ years) and included three men and two women. Two different surgeons performed electrode 
implantations irrespective of the chosen target. Surgeon A performed $3 \mathrm{STN}$ and $3 \mathrm{GPi}$ electrode implantations. Surgeon B performed four STN and two GPi electrode implantations. Table 1 recapitulates the clinical and surgical characteristics of each patient.

Patients gave clear informed consent to participate in this study and we followed strict ethical guidelines given by the Comite Consultatif National d'Ethique (The French National Review Committee).

\section{Neurosurgical procedure}

STN or GPi electrode implantations were performed with the ROSA stereotactic robot [22] (Medtech, Montpellier, France) at the University Hospital of Grenoble. Briefly, STN or GPi targeting was based on stereotactic $3 \mathrm{~T}$ magnetic resonance imaging (MRI) scan, which were imported into the planning station and reformatted into a plane parallel to the AC-PC plane by using robot planning software (ROSA from Medtech, Montpellier, France). This first step allowed determining an entry point while avoiding vessels of the cortical surface and the sulci. Then, under local anaesthesia, three to five parallel recording/stimulating microelectrodes each one into a guiding tube, and on a "BenGun" system were inserted into the brain using a motorized microdrive (Alpha-Omega, Nazareth, Israel) on a ROSA stereotactic robotic arm. The neuronal recording aimed to detect the target, based on its typical firing pattern and was followed by acute stimulation tests to determine the best site for the chronic electrode to be placed. Finally the chronic electrode (Medtronic, Minneapolis, USA) was implanted and connected to the pulse generator (Medtronic, Minneapolis, USA).

\section{Brain imprinting procedure}

Once the best location for implantation of the definitive electrode has been defined, microelectrodes were removed from the guiding tube and replaced by a $1 \mathrm{~mm}$ diameter, stainless steel blunt stylet (Dixi, Besancon, France), which slides through the guiding tube, protrudes by $1.5 \mathrm{~mm}$ out of it, and reaches the target in 10 to $15 \mathrm{~s}$ (Fig. 1a). The stylet tip was kept $1 \mathrm{~min}$ in contact with brain tissue to allow the imprinting process. To determine the location of the stylet tip into the brain during the imprint, intraoperative radiography (Fig. 1b) was performed and merged with the patient pre-operative MRI (Fig. 1c) using the robot planning software (ROSA from Medtech, Montpellier, France). The stylet was then retracted into the guiding tube so that the set can be withdrawn in 5 to $10 \mathrm{~s}$ without the stylet tip being contaminated during the removal step. Once the set had been removed from the brain, the stylet tip was immediately immersed in appropriate buffer for further analyses. From the 12 operated patients, a total of 19 different
BTI samples were made available for the present study. BTIs could not be performed in 5 hemispheres due to technical constraints.

Nineteen different brain tissue imprints (BTI) had been collected by surgeon A $(n=10)$ and $\mathrm{B}(n=9)$ and analysed by scanning electron microscopy $(n=1)$, immunohistochemistry and immunofluorescence $(n=3)$, proteomics $(n=12)$ and transcriptomics $(n=3)$ approaches. Table 1 recapitulates the type of analysis performed on each imprint.

\section{Scanning electron microscopy}

One stylet was collected in the surgical room and tissue fragments that adhered to the stylet tip was collected in fixative solution, dehydrated through an ascending serie of ethanol solution and critical point dried using $\mathrm{CO}_{2}$. Tissue fragments were sputter-coated with gold prior to examination in a Cambridge S360 scanning electron microscope.

\section{Immunohistochemistry analyses}

Stylets $(n=3)$ were collected in the surgical room and their tip was immediately immersed in fixative solution and cell block preparation was performed using the Shandon $^{\text {Tx }}$ cytoblock $^{\text {tm }}$ preparation system (Thermo Fisher Scientific, MA, USA) according to the manufacturer's protocol. Immuno-histofluorescence staining was performed on de-paraffinized BTIs $10 \mu \mathrm{m}$ thick sections using antigen retrieval treatment and with secondary antibodies coupled with Alexa fluorochrome (A488 or A555). Neuronal and astroglial expression were identified using $\beta 3$-tubulin (Covance, PRB435P) or NeuN (Millipore, MAB377) antibodies, and GFAP (DAKO, Z334) antibody, respectively. Further neuronal phenotypic characterization was investigated using antibody against: tyrosine hydroxylase (TH, Chemicon, AB152), vesicular glutamate transporter 1 for glutamatergic neurons (V-Glut1, Chemicon, MAB5502), glutamic acid decarboxylase-67 (GAD-67, Chemicon MAB5406) for GABA-ergic neurons. Sections were then counterstained with DAPI to label cell nuclei. Images were obtained using a fluorescence microscope Zeiss 5.1 (Zeiss, Iena, Germany) coupled to a digital camera and Axiovision 4.8 software (Zeiss).

\section{Proteomic analysis \\ Sample treatment}

Stylets $(n=12)$ were collected in the surgical room and the stylet tip was immersed and kept under agitation 5 min at $4{ }^{\circ} \mathrm{C}$ in urea $8 \mathrm{M} / \mathrm{CHAPS} 2 \%$ lysis buffer (Sigma-Aldrich, Saint-Louis, MO, USA). Samples were centrifuged $10 \mathrm{~min}$ at $10,000 \mathrm{~g}$ at $4{ }^{\circ} \mathrm{C}$ and the supernatant was recovered and frozen at $-80{ }^{\circ} \mathrm{C}$ until analysis. For all samples, the delay between collection, protein extraction and freezing was less than $1 \mathrm{~h}$ to prevent protein degradation. 


\section{D SDS-PAGE electrophoresis}

The total protein amount recovered from each BTI was quantified by the Bradford protein assay (Bio-Rad Laboratories, Hercules, CA, USA). Two micrograms of proteins from each BTI were individually mixed to Laemmli buffer from Bio-Rad. The mixture was heated in boiling water for $5 \mathrm{~min}$ and loaded on $15 \%$ polyacrylamide gels. Staining was performed with $0.2 \%$ silver nitrate solution for $2 \mathrm{~min}$ and protein bands were revealed in $0.02 \%$ formaldehyde/ $0.005 \%$ citric acid solution. Revelation was blocked with $1 \%$ acetic acid solution and gels were scanned.

\section{In-gel fractionation}

For both STN and GPi, $2.5 \mu \mathrm{g}$ of proteins from six different BTIs were pooled, and the resulting $15-\mu$ g total proteins were mixed to Laemmli buffer. The mixture was heated in boiling water for $5 \mathrm{~min}$ and loaded on a precast 4 to $20 \%$ polyacrylamide gel from Bio-Rad. Staining and destaining were performed with Coomassie blue and $30 \%$ ethanol/ $7 \%$ acetic acid in water respectively. STN and GPI-associated lanes were cut into 12 homogeneous fractions that were washed in $100 \mathrm{mM}$ ammonium bicarbonate for $15 \mathrm{~min}$ at $37^{\circ} \mathrm{C}$ followed by a second wash in $100 \mathrm{mM}$ ammonium bicarbonate, acetonitrile (1:1) for $15 \mathrm{~min}$ at $37^{\circ} \mathrm{C}$. Reduction and alkylation of cysteines were per- formed by mixing the gel pieces in $10 \mathrm{mM}$ DTT for $35 \mathrm{~min}$ at $56{ }^{\circ} \mathrm{C}$ followed by $55 \mathrm{mM}$ iodoacetamide for $30 \mathrm{~min}$ at room temperature in the dark. An additional cycle of washes in ammonium bicarbonate and ammonium bicarbonate/acetonitrile was then performed. Proteins were digested by incubating each gel slice with modified sequencing grade trypsin (Promega) in $50 \mathrm{mM}$ ammonium bicarbonate overnight at $37^{\circ} \mathrm{C}$. The resulting peptides were extracted from the gel by three steps: a first incubation in $50 \mathrm{mM}$ ammonium bicarbonate for $15 \mathrm{~min}$ at $37{ }^{\circ} \mathrm{C}$ and two incubations in $10 \%$ formic acid, acetonitrile (1:1) for $15 \mathrm{~min}$ at $37{ }^{\circ} \mathrm{C}$. The three collected extractions were pooled with the initial digestion supernatant, dried in a SpeedVac, and resuspended with $12 \mu \mathrm{l}$ of $5 \%$ acetonitrile, $0.1 \%$ formic acid.

\section{Nano-LC-MS/MS analysis}

Protein identification of each fraction was performed by nano-liquid chromatography tandem mass spectrometry (nano-LC-MS/MS) and each fraction was analysed twice to obtain technical replicates and to increase protein identification.

Nano-LC-MS/MS was performed on a linear trap quadrupole (LTQ) Orbitrap Velos Pro (Thermo Electron, San Jose, CA, USA) equipped with a NanoAcquity system (Waters). Peptides were trapped on a homemade $5 \mu \mathrm{m} 200 \AA$ A Magic C18 AQ (Michrom) $0.1 \times$ $20 \mathrm{~mm}$ pre-column and separated on a commercial
$0.075 \times 150 \mathrm{~mm}$ Nikkyo (Nikkyo Technology) analytical nanocolumn (C18, $5 \mu \mathrm{m}, 100 \AA$ ). The analytical separation was run for 65 min using a gradient of $\mathrm{H}_{2} \mathrm{O} / \mathrm{FA}$ $99.9 \% / 0.1 \%$ (solvent A) and $\mathrm{CH}_{3}$ CN/FA $99.9 \% / 0.1 \%$ (solvent $\mathrm{B}$ ). The gradient was run as follows: $0-1 \mathrm{~min}$ $95 \% \mathrm{~A}$ and $5 \% \mathrm{~B}$, then to $65 \% \mathrm{~A}$ and $35 \% \mathrm{~B}$ for $55 \mathrm{~min}$, and $20 \% \mathrm{~A}$ and $80 \% \mathrm{~B}$ for $65 \mathrm{~min}$ at a flow rate of $220 \mathrm{~nL} / \mathrm{min}$. For MS survey scans, the orbitrap (OT) resolution was set to 60000 and the ion population was set to $5 \times 10^{5}$ with an $\mathrm{m} / \mathrm{z}$ window from 400 to 2000 . For protein identification, up to eight precursor ions were selected for collision-induced dissociation (CID) in the LTQ. The ion population was set to $1 \times 10^{4}$ (isolation width of $2 \mathrm{~m} / \mathrm{z}$ ) while for MS/MS detection in the OT, it was set to $1 \times 10^{5}$ with an isolation width of $2 \mathrm{~m} / \mathrm{z}$ units. The normalized collision energies were set to $35 \%$ for CID.

\section{Peptide and protein identification}

MS data were processed using EasyProtConv. For each imprinted nucleus, the peak list of each fraction was generated from raw data CID spectra using the EasyProtConv conversion module. The peak lists of all fractions were then merged into a single peak list file per nucleus on EasyProtConv. The two different obtained peak lists were submitted to Easyprot, a platform that uses Phenyx (GeneBio, Geneva, Switzerland) for protein identification [39]. Searches were conducted against UniProt Swiss-Prot database (669 903 entries on October, 2014) specifying Homo sapiens taxonomy. Specificity of trypsin digestion was set for cleavage after Lys or Arg except before Pro, and two missed trypsin cleavages were allowed. Carbamidomethylation of cysteines was set as a fixed modification, and oxidation of methionine was set as variable modifications. Peptide z-scores were then set to maintain a false positive peptide ratio below $1 \%$. Proteins with at least two distinct peptide sequences were automatically validated. Proteins were clustered based on shared peptides, with the protein entry containing the most peptides selected as the group reporter.

\section{Functional analysis by ingenuity pathway analysis}

The STN and GPI-associated protein lists were further submitted to Ingenuity Pathway Analysis software (Ingenuity Systems, Redwood City, CA) to perform the functional characterization of the proteins recovered from the BTIs. This automatic annotation tool uses a knowledge database and assigns proteins to functional classes or specific canonical pathways $(\mathrm{CPs})$ related to various biological processes [23].

\section{Transcriptomic analysis}

\section{RNA extraction, amplification and quality control}

STN-associated BTIs $(n=3)$ were collected in the surgical room and quickly immersed and kept under agitation 
$5 \mathrm{~min}$ in $100 \mu \mathrm{l}$ lysis buffer from the mirVana isolation kit $^{\text {is }}$ (Ambion, Applied Biosystems, Foster City, CA) to prevent RNase activity and therefore to limit RNA degradation. In parallel, two $60 \mu \mathrm{m}$-thick sections from a cortical piece, which was obtained during a previous surgical tissue resection in an epilepsy patient, were cut at $-20{ }^{\circ} \mathrm{C}$ and immediately immersed in $100 \mu \mathrm{l}$ lysis buffer. This sample was used as a technical control. After tissue lysis, total RNA was isolated from BTI and technical control using a phenol:chloroform extraction protocol (MirVana isolation $\mathrm{kit}^{\mathrm{sm}}$, Ambion, Applied Biosystems). Before RNA amplification, the quality and integrity of the technical control were confirmed (RNA integrity $=8.3$, suppl data 2 ) by capillary electrophoresis migration using RNA nano 6000 kit and the Bioanalyzer 2100 (Agilent Technologies, Palo Alto, CA). Then total RNAs from BTIs and technical control were separately amplified with the GeneChip 3' IVT Express Kit. First, total RNAs were reverse-transcribed using primers polydT-T7 promoter sequence; cDNAs were double-stranded synthesized and finally amplified by the T7 RNA polymerase. A second amplification was performed with WT-expression kit $^{\text {Ti }}$ (Ambion, Applied Biosystems, Foster City, CA) to obtain biotinylated cDNA. After this double amplication procedure, the RNA concentration of each BTI sample was determined with the measure of absorbance at $260 \mathrm{~nm}$, and the electrophoretic profiles of BTI-samples were compared to the technical control to indirectly confirm the quality of BTI-extracted RNA.

\section{Microarray hybridization}

Microarrays experiments were carried out in accordance with the protocol GeneChip Expression Wash, Stain and Scan from Affymetrix (Santa Clara, CA, USA). Briefly, $15 \mu \mathrm{g}$ of labeled cRNA was hybridized for $16 \mathrm{~h}$ at $45^{\circ} \mathrm{C}$ on GeneChip Human Genome U133 Plus 2.0 (Affymetrix, Santa Clara, CA, USA) corresponding to 54675 unique gene probe sets. The arrays were washed and stained with streptavidin - phycoerythrin before scanning. The fluorescence values of each probe set signal, reported in arbitrary units, were processed with MAS5 statistical algorithm to validate the probe set signal "absent" or "present", and finally they were normalized between all the arrays using the Robust Multichip Analysis (RMA) algorithm. These algorithms are included in Affymetrix expression console (Affymetrix, Santa Clara, CA, USA).

\section{Additional files}

Additional file 1: Figure S1. BTI-associated immunohistochemical analyses. Immunostaining of the dilator-bound material (X200) revealed (top) the presence of many cells by hematoxylin-eosin staining among which (middle) neurons by neuronal nuclei (NeuN) staining and (bottom) astrocytes by glial fibrillary acid protein (GFAP) staining. (DOCX 551 kb)
Additional file 2: Table S1. Non-redundant lists of the proteins identified in STN or GPi-associated BTIs. (XLSX 401 kb)

Additional file 3: Figure S2. Confirmation of the technical controlrelated RNA Integrity by capillary electrophoresis. (DOCX 191 kb)

Additional file 4: Table S2. Micro-array hybridization results. The table recapitulates detected $(P)$ and non-detected $(A)$ transcripts in all analyzed samples. (XLSX $12765 \mathrm{~kb}$ )

\section{Competing interests}

The authors declare that they have no competing interests.

\section{Authors' contributions}

AZ designed BTI experiments, treated BTIs, performed proteomics experiments, analysed data and wrote the manuscript. AB designed BTI experiments, treated BTIs, analysed data and wrote the manuscript. SC performed electrode implantation, collected BTls and wrote the manuscript. MEA performed and analysed transcriptomics experiments. ES performed electrode implantation and collected BTIs. JAL designed and analysed immunohistochemistry experiments. MDD designed, performed, analysed immunohistochemistry experiments and wrote the manuscript. FB designed the BTI experiments, funded transcriptomics experiments and wrote the manuscript. PB designed BTI experiments, analysed proteomics data, wrote the manuscript and funded proteomics experiments. All authors read and approved the final manuscript.

\section{Acknowledgments}

Parkinson Swiss, Swiss National Funding, Region Rhône-Alpes, EDF Foundation supported this work.

\section{Author details}

${ }^{1}$ NeuroProteomics Group, University Medical Center, Faculty of Medicine, Geneva, Switzerland. ${ }^{2}$ CEA-LETI, Clinatec, Edmond J. Safra Biomedical Research Center, Grenoble, France. ${ }^{3}$ Clinique de neurochirurgie, CHU de Grenoble, Grenoble, France. ${ }^{4}$ Department of Pathology, Geneva University Hospitals, Geneva, Switzerland. 5 Department of Pathology and Immunology, University Medical Center, Faculty of Medicine, Geneva, Switzerland. ${ }^{6}$ Inserm UA01, Clinatec, Edmond J. Safra Biomedical Research Center, CEA, Grenoble CHU, Univ. Grenoble Alpes, Grenoble, France. ${ }^{7}$ Inserm, U836, Grenoble Institut des Neurosciences, Univ. Grenoble Alpes, Grenoble, France.

${ }^{8}$ Department of Neurology, Geneva University Hospitals, Geneva, Switzerland.

${ }^{9}$ Centre Medical Universitaire (CMU), Rue Michel Servet 1, CH-1211 Genève 4, Switzerland.

Received: 21 September 2015 Accepted: 20 January 2016

Published online: 28 January 2016

\section{References}

1. Lang AE, Lozano AM. Parkinson's disease. First of two parts. N Engl J Med. 1998:339(15):1044-53.

2. Lang AE, Lozano AM. Parkinson's disease. Second of two parts. N Engl J Med. 1998;339(16):1130-43.

3. Samii A, Nutt JG, Ransom BR. Parkinson's disease. Lancet. 2004;363(9423): 1783-93.

4. Olanow CW, Tatton WG. Etiology and pathogenesis of Parkinson's disease. Annu Rev Neurosci. 1999;22:123-44.

5. Potashkin JA, Blume SR, Runkle NK. Limitations of animal models of Parkinson's disease. Parks Dis. 2010;2011:658083.

6. Lin MT, Beal MF. Mitochondrial dysfunction and oxidative stress in neurodegenerative diseases. Nature. 2006;443(7113):787-95.

7. McNaught KS, Olanow CW, Halliwell B, Isacson O, Jenner P. Failure of the ubiquitin-proteasome system in Parkinson's disease. Nat Rev Neurosci. 2001; 2(8):589-94.

8. Licker V, Burkhard PR. Neuroproteomics and Parkinson's disease: don't forget human samples. Expert Rev Proteomics. 2011;8(3):291-4.

9. International Parkinson Disease Genomics C, Nalls MA, Plagnol V, Hernandez DG, Sharma M, Sheerin UM, et al. Imputation of sequence variants for identification of genetic risks for Parkinson's disease: a meta-analysis of genome-wide association studies. Lancet. 2011;377(9766):641-9. 
10. Nalls MA, Pankratz N, Lill CM, Do CB, Hernandez DG, Saad M, et al. Largescale meta-analysis of genome-wide association data identifies six new risk loci for Parkinson's disease. Nat Genet. 2014;46(9):989-93.

11. Cooper-Knock J, Kirby J, Ferraiuolo L, Heath PR, Rattray M, Shaw PJ. Gene expression profiling in human neurodegenerative disease. Nat Rev Neurol. 2012:8(9):518-30.

12. Zhang J, Keene CD, Pan C, Montine KS, Montine TJ. Proteomics of human neurodegenerative diseases. J Neuropathol Exp Neurol. 2008;67(10):923-32.

13. Caudle WM, Bammler TK, Lin Y, Pan S, Zhang J. Using 'omics' to define pathogenesis and biomarkers of Parkinson's disease. Expert Rev Neurother. 2010;10(6):925-42

14. Licker V, Kovari E, Hochstrasser DF, Burkhard PR. Proteomics in human Parkinson's disease research. J Proteome. 2009;73(1):10-29.

15. Ravid R, Ikemoto K. Pitfalls and practicalities in collecting and banking human brain tissues for research on psychiatric and neulogical disorders. Fukushima J Med Sci. 2012;58(1):82-7

16. Stan AD, Ghose S, Gao XM, Roberts RC, Lewis-Amezcua K, Hatanpaa KJ, et al. Human postmortem tissue: what quality markers matter? Brain Res. 2006; 1123(1):1-11.

17. Preece $P$, Cairns NJ. Quantifying mRNA in postmortem human brain: influence of gender, age at death, postmortem interval, brain $\mathrm{pH}$, agonal state and interlobe mRNA variance. Brain Res Mol Brain Res. 2003;118(1-2):60-71.

18. Chariot P, Witt K, Pautot V, Porcher R, Thomas G, Zafrani ES, et al. Declining autopsy rate in a French hospital: physician's attitudes to the autopsy and use of autopsy material in research publications. Arch Pathol Lab Med. 2000; 124(5):739-45.

19. Ferrer I, Santpere G, Arzberger T, Bell J, Blanco R, Boluda S, et al. Brain protein preservation largely depends on the postmortem storage temperature: implications for study of proteins in human neurologic diseases and management of brain banks: a BrainNet Europe Study. J Neuropathol Exp Neurol. 2007;66(1):35-46.

20. Tomita H, Vawter MP, Walsh DM, Evans SJ, Choudary PV, Li J, et al. Effect of agonal and postmortem factors on gene expression profile: quality control in microarray analyses of postmortem human brain. Biol Psychiatry. 2004; 55(4):346-52.

21. Benabid AL. Deep brain stimulation for Parkinson's disease. Curr Opin Neurobiol. 2003;13(6):696-706.

22. Lefranc M, Le Gars D. Robotic implantation of deep brain stimulation leads, assisted by intra-operative, flat-panel CT. Acta Neurochir. 2012;154(11):2069-74.

23. Jimenez-Marin A, Collado-Romero M, Ramirez-Boo M, Arce C, Garrido Ju. Biological pathway analysis by ArrayUnlock and Ingenuity Pathway Analysis. BMC Proc. 2009;3 Suppl 4:S6.

24. Benabid AL, Pollak P, Louveau A, Henry S, de Rougemont J. Combined (thalamotomy and stimulation) stereotactic surgery of the VIM thalamic nucleus for bilateral Parkinson disease. Appl Neurophysiol. 1987;50(1-6):344-6.

25. Benabid AL, Chabardes S, Mitrofanis J, Pollak P. Deep brain stimulation of the subthalamic nucleus for the treatment of Parkinson's disease. Lancet Neurol. 2009:8(1):67-81.

26. Kumar R, Lang AE, Rodriguez-Oroz MC, Lozano AM, Limousin P, Pollak P, et al. Deep brain stimulation of the globus pallidus pars interna in advanced Parkinson's disease. Neurology. 2000;55(12 Suppl 6):S34-9.

27. Plaha P, Gill SS. Bilateral deep brain stimulation of the pedunculopontine nucleus for Parkinson's disease. Neuroreport. 2005;16(17):1883-7.

28. Blandini F, Nappi G, Tassorelli C, Martignoni E. Functional changes of the basal ganglia circuitry in Parkinson's disease. Prog Neurobiol. 2000;62(1):63-88.

29. Cossette $M$, Levesque $M$, Parent $A$. Extrastriatal dopaminergic innervation of human basal ganglia. Neurosci Res. 1999;34(1):51-4.

30. Schuepbach WM, Rau J, Knudsen K, Volkmann J, Krack P, Timmermann L, et al. Neurostimulation for Parkinson's disease with early motor complications. N Engl J Med. 2013;368(7):610-22.

31. Perlmutter JS, Mink JW. Deep brain stimulation. Annu Rev Neurosci. 2006;29:229-57.

32. Holtzheimer PE, Mayberg HS. Deep brain stimulation for psychiatric disorders. Annu Rev Neurosci. 2011;34:289-307.

33. Ramayya AG, Zaghloul KA, Weidemann CT, Baltuch GH, Kahana MJ. Electrophysiological evidence for functionally distinct neuronal populations in the human substantia nigra. Front Hum Neurosci. 2014;8:655
34. Mallet L, Polosan M, Jaafari N, Baup N, Welter ML, Fontaine D, et al Subthalamic Nucleus Stimulation in Severe Obsessive-Compulsive Disorder. New Engl J Med. 2008;359(20):2121-34.

35. Chabardes S, Kahane P, Minotti L, Koudsie A, Hirsch E, Benabid AL. Deep brain stimulation in epilepsy with particular reference to the subthalamic nucleus. Epileptic Disord. 2002;4 Suppl 3:S83-93.

36. Vercueil L, Pollak P, Fraix V, Caputo E, Moro E, Benazzouz A, et al. Deep brain stimulation in the treatment of severe dystonia. J Neurol. 2001;248(8):695-700.

37. Shahed J, Poysky J, Kenney C, Simpson R, Jankovic J. GPi deep brain stimulation for Tourette syndrome improves tics and psychiatric comorbidities. Neurology. 2007;68(2):159-60.

38. Benabid AL, Pollak P, Gervason C, Hoffmann D, Gao DM, Hommel M, et al Long-term suppression of tremor by chronic stimulation of the ventral intermediate thalamic nucleus. Lancet. 1991:337(8738):403-6.

39. Gluck F, Hoogland C, Antinori P, Robin X, Nikitin F, Zufferey A, et al. EasyProt-an easy-to-use graphical platform for proteomics data analysis. J Proteome. 2013;79:146-60.

\section{Submit your next manuscript to BioMed Central and we will help you at every step:}

- We accept pre-submission inquiries

- Our selector tool helps you to find the most relevant journal

- We provide round the clock customer support

- Convenient online submission

- Thorough peer review

- Inclusion in PubMed and all major indexing services

- Maximum visibility for your research

Submit your manuscript at www.biomedcentral.com/submit 